\title{
A new method to predict adult-onset type 1 diabetes
}

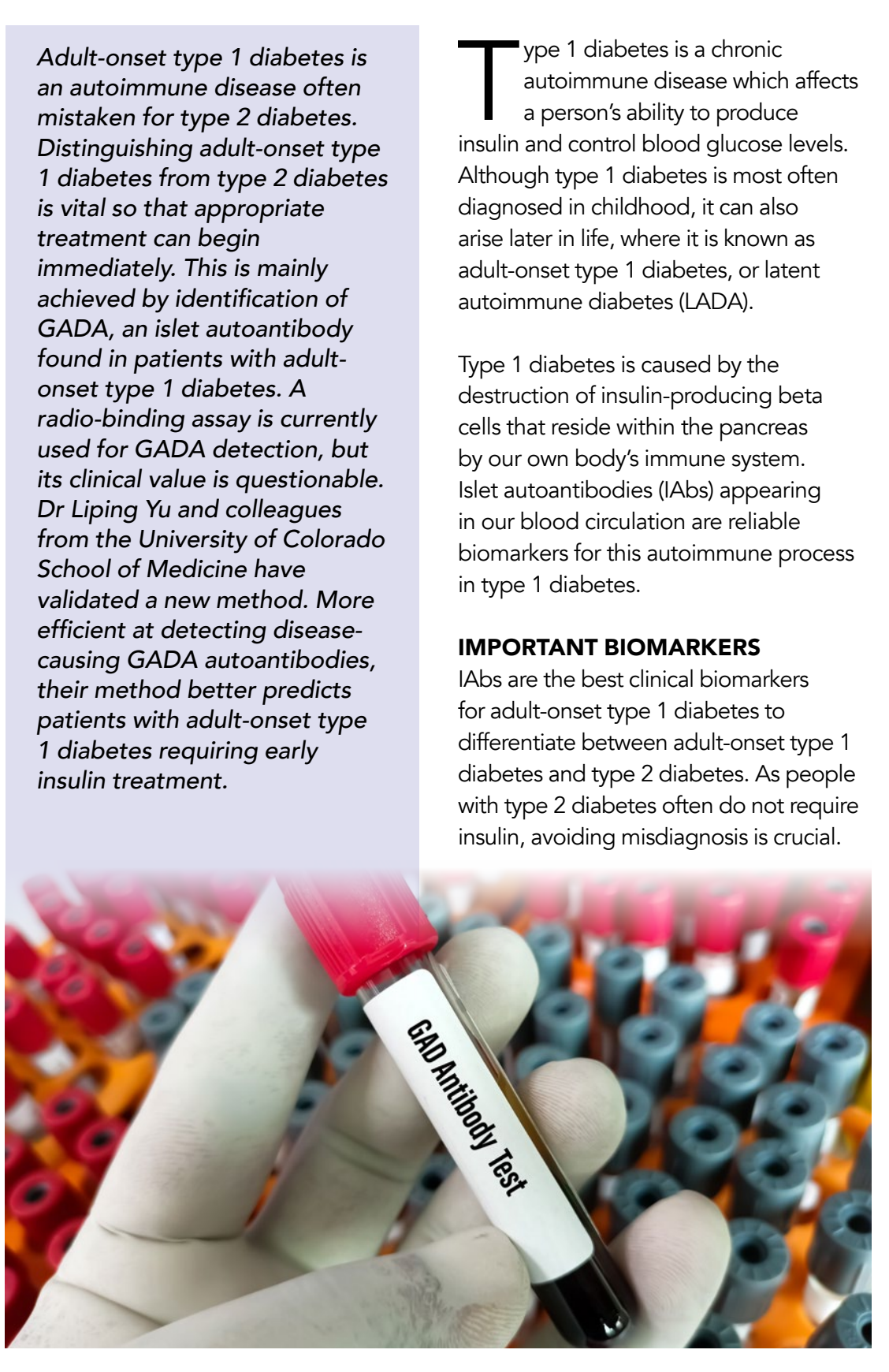

Glutamic acid decarboxylase (GAD) 65 (GADA) are the most are often the only diabetes sinceciated auto 1 diabetes.

CURRENT CLINICAL DIAGNOSIS Radio-binding assays (RBA) are the standard detection method for measuring levels of GADA for predicting adult-onset type 1 diabetes. These RBA-GADA use radioactive tracers that bind to the GADA. If GADA are present in a patient's sample, a positive result is identified by a radioactive signal measured in the sample, and the level of radioactivity is proportional to the mount of antibody present.

Unfortunately, RBA-GADA generates a lot of biologically false positives, or in -ility to distinguish be due to ts and low-affinity binding GADA. This is important for screening since low-disease risk autoantibodies can be present in the patient's blood but are not associated with type 1 diabetes. Although RBAGADA demonstrates high specificity to detect autoantibodies (98-99\%) biochemically, in terms of disease, the overall accuracy of clinical diagnosis is low-affinity GADA generated.

\section{A NEW TOOL}

To overcome these shortfalls in GADA screening, Dr Yu and colleagues from the University of Colorado Schood extensively validated a non-radioactive



Not only is the ECL-GADA assay low-cost and non-radioactive, it detects clinicallyrelevant high-affinity binding GADA and other islet autoantibodies.

electrochemiluminescence (ECL) assay to accurately screen for adult-onset type 1 diabetes. Their ECL assay has been shown to be as sensitive as RBA but importantly, it possesses a higher-aftinity autoantibody
detection of GADA, effectively removing
low-affinity signals (false positives). In their study, 2834 patient were obtined from two patient samples that had been enrolled in larger clinical studies: Action LADA in the UK laged 30-70 years), and DiYA in the USA (aged 20-45 years). In both studies, individuals were diagnosed with adult-onset diabetes within five years, and some had early insulin therapy.

In the Action LADA study, 278 patients were positive for RBA-GADA with or without other autoantibodies, including insulinoma-associated antigen 2 (IA-2A) or zinc transporter-8 autoantibodies (ZnT8A), and 493 patients were negative In the DiA study, 25 individuals were positive for RBA.GADA wh orw were negative. RBA for GADA, IA-2A, and Zing all the clinic study samples.

GADA IS THE DOMINANT AUTOANTIBODY IN ADULTONSET DIABETES LADA study) and 6.6\% IAbs positive (DiYA study). In both groups, RBA-GADA was the most dominant and prevalent $\mid A b$, seen over $90 \%$ of the time. Other IAbs (IA-2A and ZnT8A) were observed, but only in a small fraction of individuals. Most individuals with adult-onset type 1 diabetes were single positive GADA without other IAbs (A-2A and ZnT8A): in the DiYA study.

In both groups, ECL-GADA positivity was more associated with participants that had multiple autoantibodies. Interesting positivity was only seen in $62 \%$ with RBA-
Of the total adult-onset diabetes patients,
GADA alone, compared to participants with more than one autoantibody (eg, GADA plus IA-2A and/or ZnT8A (87\%)). In the DiYA study, ECL-GADA positivity with RBA-GADA alone, and in 100\% of participants with RBA-GADA plus IA-2A and/or ZnT8A.

DIFFERENTIATING BETWEEN ADULTONSET TYPE 1 DIABETES AND TYPE 2 DIABETES

Importantly, detection of GADA by RBA TCL assays could differentiate betwe diabetes and type 2 diabetes:

Participants who were GADA positive also significantly leaner, more frequently positive for multiple IAbs (IA-2A and Znt8A), and often required early insulin which were GADA positive for both assays were significantly more likely to require early insulin treatment compared to participants who were negative for GADA in both assays. Interestingly, participants in both the younger study (DiYA study) and the older study (Acton LADA) with a low body mass index (BM) with adult-onset in both assays compared to those negative in both GADA assays (which presumably had type 2 diabetes) were treatment. Participants in both cohorts 
early insulin treatment, independen of GADA positivity. Highlighting the alongside screening is important as it aids disease prediction.

ECL ASSAY IS MORE SPECIFIC FO ADULT-ONSET TYPE 1 DIABETES Across both cohorts, RBA-GADA detected a higher number of positive assay. In the Action LADA cohort, 278 out of 771 individuals were RBA-GADA positive, but only 188 participants of these were ECL-GADA positive. Similarly, in the DiYA cohort, 125 out of 2,063 were RBA-GADA positive and 93 of these were positive for ECL-GADA. Overall, $26 \%$ (DiYA study) and $32 \%$ (Action LADA) of GADA detected by RBA were previous findings that This aligns with f single GADA positivity by RBA in type 1 diabetes-screening RBA in population or first-degree relatives of patients with type 1 diabetes were confirmed by ECL assay. This shows that there is a clear discrepancy between the two screening methods.

Interestingly, participants with RBA-GADA positivity but not ECL-GADA displayed similar characteristics to type 2 diabetes across both cohorts. These participants were more obese, less likely to have multiple autoantibodies, and were less likely to require early insulin treatm Yu and team found that multiple

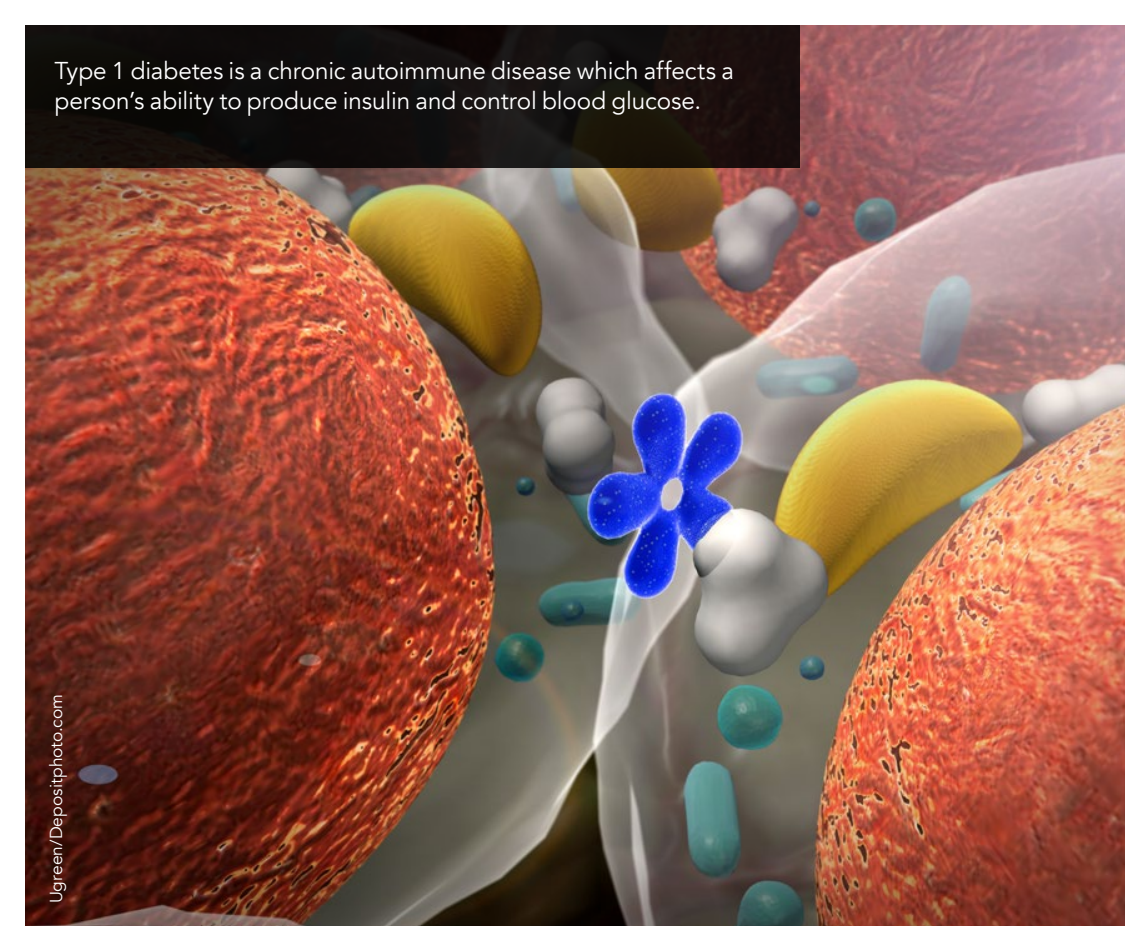

by the requirement of a higher level of GAD65 protein for the absorption samples, compared to ECL-assay-pos samples. It also confirms a lower affinity of GADA detected by RBA were less or not predictive, and supports previous findings that the ECL-GADA assay detects higher affinity GADA, and is therefore a more accurate predictor of adult-onset type 1 diabetes.

Higher affinity GADA was a predictor for early insulin requirement too, but Yu's

To overcome shortfalls in GADA autoantibody screening, Dr Yu and colleagues have developed and extensively validated an assay to accurately screen for adult-onset type 1 diabetes.

autoantibodies did not increase the risk of requiring early insulin

GADA positive patients.

ECL ASSAY DISCRIMINATES LOWAFFINITY AUTOANTIBODIES GADA-affinity testing confirmed that GADA autoantibodies confirmed by the ECL assay were of higher affinity by RBA-GADA alive. This is hightighted clinical characteristics alone were a significant predictor of this, independen of GADA positivity. This was seen in individuals across both study groups with an earlier age diagnosis and a lower BM. ECL-GADA positivity and BMI <25kg/ $m^{2}$ helped predict requirement for early insulin from $37.4 \%$ to $45.7 \%$ in the DiYA cohort, and from $40.8 \%$ to $55.3 \%$ in the first degree relatives of patients with type witicitipan who were RBA-GADA positive but negative for ECL-GADA have unchanged normal blood glucose during a 4.7 year by ECL-GADA had dysregulated blood glucose comparable to high type diabetes risk subjects with multipl islet autoantibodies. These findings suggest that both the ECL-GADA assay and clinical characteristics are strong predictors of adult-onset type 1 diabetes and early insulin treatment.

\section{CLINICAL IMPORTANCE}

Yu's study highlights the importance mentancing current clinical screening

assay low-cost and non-radioactive (unlike

the RBA), it detects clinically-relevant high-affinity binding GADA and other islet autoantibodies. The new ECL assay thus offers a better prediction of adultonset type 1 diabetes requiring early insulin treatment compared to current be significantly valuable to aid screening and should be further considered for determining individuals at risk for adultonset type 1 diabetes. Importantly, the CCL-GADA assay could improve routine leading to a more accurate dites, leading to a more accurstect follow up. However, in participants who were RBA-GADA positive and confirmed screening methods. Yu's studies also suggest that patient demographics could

\section{ब. Behind the Research}

il Dr Liping Yu

E: liping.yu@cuanschutz.edu T: +1 303-724-6808 W: www.barbaradaviscenter.org

Research Objectives

Liping Yu's long-term research interests are mainly focused on clinical immunology and the genetics of type 1 diabetes.

\section{Detail}

Address

Barbara Davis Center for Diabetes

CO 80045, USA

Bio

Dr Liping Yu is associate professor at Barbara Davis Center for Diabetes University of Colorado School of Medicine/ and Director of NIH/NIDDK designated Autoantibody/ HLA reference Laboratory His most recent studies are directly connected to multiple clinical trials that aim to understand the immunogenetics and immunopathogenesis of type 1 diabetes.

\section{Funding}

- NDRF

- NIH/NIDDK

- $\mathrm{CDC}$

The 5th Framework Program of the European Union

Collaborators

Drid Leslie and Dr Jean Lawrence, the Action LADA Diabetes in Young Adults (DiYA) Study Group

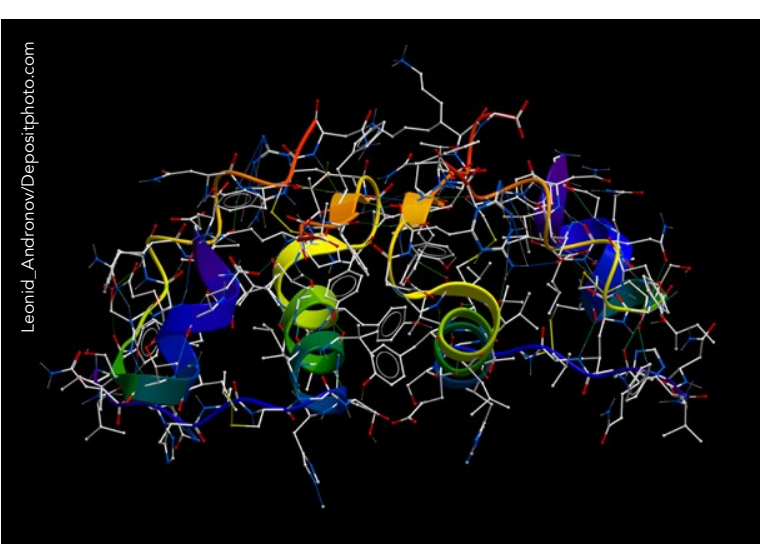

\section{References}

Gu, Y, Jia, X, Vartak, T, et al, and the Action LADA consortium and the Diabetes in Young Adults (DiYA) Study group

(2021) Improving clinical utility of GAD65 autoantibodies by electrochemiluminescence assay and clinical phenotype when $64,2052-2060$. do: 10 adut-onset diabetes. Diabetologica. 64, 2052-2060. doi:10.1007/s00125-021-05492-6

He, L, Jia, X, and Yu, L, (2021) Islet Autoantibodies, Assay Specificity and Disease Specificity. Austin Journal of Endocrinology and Dia Miao, D, Steck, AK, Zhang, L, et al, (2015) Type 1 Diabetes for insulin and glutamic acid decarboxylase autoantibodies improve prediction of type 1 diabetes risk. Diabetes Technol Ther, 17(2):119-127. doi.org/10.1089/dia.2014.0186

Miao, D, Guyer, KM, Dong, F, et al, (2013) GAD65 autoantibodies detected by electrochemiluminescence assay identify high risk for type 1 diabetes. Diabetes 62(12):41744178. doi.org/10.2337/db13-0534

\section{Personal Response}

Could a similar electrochemiluminescence assay be used to screen for childhood type 1 diabetes or other autoimmune diseases?

II ECL assay has been used for all four islet autoantibodies and also autoantibodies to other autoimmune diseases like autoantibodies to tissue transglutaminase (TGA) for celiac disease, to thyroid peroxidase (TPO) and thyroid globulin (ThG) for autoimmune thyroid disease. With the ECL assay platform, a multiplex ECL assay has been developed to combine up to ten autoantibody tests in one single tube. The multiplex ECL assay provides a high-affinity antibody for large population screening of diseases simultaneously. 